\title{
Beneficial use of immunoglobulins in the treatment of Sydenham chorea
}

\author{
Tabitha D. van Immerzeel • Ruud M. van Gilst • \\ Nico G. Hartwig
}

Received: 28 January 2010 / Accepted: 15 February 2010 /Published online: 30 March 2010

(C) The Author(s) 2010. This article is published with open access at Springerlink.com

\begin{abstract}
This double case report indicates that treatment with intravenous immunoglobulins (IVIG) is effective in patients with Sydenham chorea (SC). SC is a rare but impressive clinical manifestation following streptococcal infection. This movement disorder characterised by chorea, emotional lability and muscle weakness, is one of the major criteria of acute rheumatic fever. Treatment of SC is typically limited to supportive care and palliative medications. Curative treatment is still in the experimental stage. Recent research on patients with SC proved that antibodies against the group A streptococcus cross-react with epitopes of neurons in the basal ganglia, namely, intracellular tubulin and extracellular lysoganglioside. Therefore, immune modulating therapy by means of prednisone, plasma exchange and IVIG are mentioned in the literature as possible effective treatment. Beneficial effect of IVIG has been shown in several diseases with molecular mimicry as the underlying pathophysiology. In this paper, we describe two girls aged 11 and 13 years, respectively, who presented with SC having severe disabilities in their daily live. We treated both patients with IVIG $400 \mathrm{mg} / \mathrm{kg} /$ day for 5 days. Treatment was tolerated well and had a pronounced positive
\end{abstract}

T. D. van Immerzeel $\cdot$ R. M. van Gilst

Paediatric Department, Van Weel Bethesda Ziekenhuis,

Stationsweg 22,

3247 BW Dirksland, The Netherlands

\section{N. G. Hartwig}

Infectious Disease and Immunology Department,

Erasmus Medical Centre,

P.O. Box 2040, 3000 CA Rotterdam, The Netherlands

T. D. van Immerzeel $(\bowtie)$

Bonaventurastraat 57A1,

3081 HB Rotterdam, The Netherlands

e-mail: tabithakieviet@gmail.com effect. Shortly after the drug was administered, all signs and symptoms disappeared in both patients. Based upon these patients, we highlight IVIG as a serious treatment option for SC.

Keywords Sydenham chorea $\cdot$ St. Vitus's dance . Rheumatic fever - Streptococcal infections .

Intravenous immunoglobulins · Molecular mimicry
Abbreviations
SC Sydenham chorea
ARF Acute rheumatic fever
IVIG Intravenous immunoglobulins
ESR Erythrocyte sedimentation rate
ASOT Antistreptolysin O titre
ABGA Anti-basal ganglia antibodies

\section{Introduction}

Sydenham chorea (SC), also known as St. Vitus dance and chorea minor, is a rare but impressive clinical manifestation following streptococcal infection. This movement disorder characterised by chorea, emotional lability and muscle weakness is one of the major criteria of acute rheumatic fever (ARF) [24]. Chorea is observed in $18-36 \%$ of all patients with ARF [3, 25, 29].

The incidence of ARF and SC reflects the adequacy of preventive medical care in a community and has declined dramatically in the Western world $[9,20]$. However, chorea is still a common manifestation of ARF in developing countries, and a number of recent outbreaks in the USA indicate the need for awareness of this disease [3, 25]. 
The natural course of the illness varies widely. Complaints can last for months to years, and $20-42 \%$ of the patients have a relapse [8, 16]. Treatment of SC is typically limited to supportive care and palliative medications. Curative treatment is still in the experimental stage $[4,7,30]$.

In this paper, we describe two patients with $\mathrm{SC}$ and severe disabilities in daily life. Based upon the experience with them, we advocate the use of intravenous immunoglobulins (IVIG) as a treatment option for SC in view of the latest insights in the pathophysiology of SC.

\section{Patients}

Patient number 1, an 11-year-old girl, presented at the adult outpatient neurology department with involuntary movements since several days, which she described as "My arms hit and my legs kick while I do not want them to do so". Her parents expressed concern about garbled speech, emotional lability and regressive behaviour. There were no indications for recent infections. Especially, sore throat, joint pain and fever were not noticed. However, she did complain about general muscle weakness.

On physical examination, we saw a girl with a figure fitting her age showing continual irregular, uncontrolled movements of the upper and lower extremities and to some extend also of the head and trunk. Her speech was incomprehensive and, at rest, she made sucking and swallowing movements. Coordination was intact, illustrated by the ability to catch a ball despite her chaotic movements. There were no further neurological abnormalities. Further examination revealed enlarged, painless submandibular lymph nodes. There were no a clinical signs of endocarditis.

Laboratory findings showed a normal complete blood cell count, erythrocyte sedimentation rate (ESR) of $6 \mathrm{~mm} /$ $\mathrm{h}(1-25 \mathrm{~mm} / \mathrm{h})$ and an antistreptolysin O titre (ASOT) of $800-1,600 \mathrm{IU} / \mathrm{ml}(<250 \mathrm{IU} / \mathrm{ml})$. Rheumatoid factor and anticardiolipin antibody were negative. In the throat culture, performed several weeks after presentation, no pathogens were isolated. Computed tomography of the brain did not reveal any anatomical anomaly.

A penicillin course of treatment was given. Concerning the SC, a policy of "wait and see" was followed because of the well-known high number of patients who recover spontaneously. However, after 2 months, her symptoms had worsened, and she became wheelchair bound. Subsequently, she was referred to us.

Based upon analyses of recent literature (see below), we treated her with IVIG: $400 \mathrm{mg} / \mathrm{kg}$ per day for 5 days. Treatment was tolerated well and had a pronounced positive effect on clinical symptoms. Within several days, her mobility increased, and she was no longer wheelchair bound. Within 1 week, the SC disappeared fully. A prophylactic regimen of penicillin was administered, and in a follow-up period of 2 years, no relapse of the disease occurred.

Patient number 2, a 13-year-old girl, was referred to us by her physiotherapist because of dysarthria, ataxia and balance problems. This child was initially referred for physiotherapy because of chronic fatigue.

At referral, the complaints existed for 2 weeks and were slowly progressive. She had severe balance difficulties, made involuntary movements with her mouth and tongue and suffered from general muscle weakness. A month before, she had been diagnosed with pneumonia for which her general practitioner had treated her with amoxicillin/ clavulanate.

On physical examination, the patient made involuntary oral movements smacking her lips and moving her tongue in and out of her mouth. Examination of the larger muscle groups did not show evident muscle weakness. Her reflexes were normal, but her coordination was slightly abnormal with a deviant tandem walk. Internal examination revealed a holosystolic cardiac murmur grade $2 / 6$ with punctum maximum at the apex.

Additional laboratory research showed a normal blood cell count, ESR of $29 \mathrm{~mm} / \mathrm{h}$ and an ASOT of $>1,600 \mathrm{IU} / \mathrm{ml}$. In a throat culture, a group A haemolytic streptococcus was found. Brain magnetic resonance imaging was normal. A cardiac ultrasound revealed a mitralis valve insufficiency without hemodynamic instability.

The patient was given IVIG similarly to patient A. After administering the drug, the complaints improved substantially. The chorea disappeared completely within days. The same prophylactic penicillin regimen was used, and for the corvitium, carbasalate calcium was prescribed. Fatigue persisted for some weeks. Until now, 2 years later, this girl also did not have any relapse.

\section{Pathophysiology}

The relation between $\mathrm{SC}$ and a previous group A streptococcal (GAS) infection has been established in 1956 [27]. In 1976, Husby et al. discovered in serum of SC patients that antibodies directed against part of the GAS bacterium cross-react with neurons of the caudate nucleus and basal ganglia, a mechanism called molecular mimicry [12]. Such a pathophysiologic mechanism is also present in other diseases, for example, in Guillain-Barré syndrome, where antibodies cross-react with the antiganglioside $\mathrm{AB}$ on motor neurones and in idiopathic thrombocytopenic purpura (ITP) with glycoprotein IIbIIIa on platelets $[21,26]$. 
The GAS has two potential epitopes capable of crossreactivity. $\mathrm{N}$-acetyl-beta-D-glucosamine (GlcNAc) is a structural component of the streptococcal cell wall and is the epitope that actually provokes the antibodies that play a role in some manifestations of ARF [15, 17]. Several studies show that GlcNAc cross-reacts with glycoconjugates of different molecular composition, for example, glycoproteins on cardiac valve surface in rheumatic heart disease. A second molecule called $\mathrm{M}$ protein, the major virulence factor of GAS, turned out to be cross-reactive with various host $\alpha$-helical proteins $[6,15]$.

Bronze and Dale continued to elucidate the pathogenesis of SC by discovering anti-basal ganglia antibodies (ABGA) [2]. They demonstrated that rabbits immunised with the group A M protein produced antiserum that was crossreactive with central nerve system antigens in Western blots. Reactivity to those specific neuronal antigens was inhibited by pre-incubation with GAS carbohydrate [2]. It was made clear that these ABGA have a high specificity and sensitivity [6]. The specific neuronal target antigens in SC were only recently discovered by Kirvan et al. [14]. They studied human antibodies derived from serum of an SC patient and found cross-reactivity with intracellular brain protein tubulin and extracellular lysoganglioside. By binding to those proteins, a signalling cascade was triggered that may cause the neurological manifestations of SC by releasing dopamine to the synapse [14].

\section{Discussion}

Textbooks suggest symptomatic treatment for SC with benzodiazepine, anti-epileptics, phenothiazines or neuroleptics. These drugs are central nerve system depressants and control the main neurological signs of SC. The effect of these drugs is variable $[7,11,23]$.

The pathophysiology of cross-reactive antibodies in SC implies new treatment options supported by experiences with immune and inflammatory disorders described above. Indeed, immune modulating therapy, like corticosteroids or immunoglobulin infusion, is found to improve SC.

Corticosteroids have been studied most extensively. There is only one randomised double blind study in which 22 children suffering from SC were treated with $2 \mathrm{mg} / \mathrm{kg} /$ day prednisone during 4 weeks followed by a gradual discontinuation. On evaluation in weeks 8 and 12, these patients showed a significant reduction in chorea intensity compared with a 15 patient placebo group [22]. Several other studies (case reports or retrospective cohorts) demonstrate that prednisone improves the course of the disease $[1,5,28]$. However, many of these reports note a rapid relapse of symptoms or the development of important side effects like Cushing's syndrome and hypertension.
In the past decade, IVIG have been increasingly used in the treatment of diseases caused by auto-antibodies [13, 31]. The effect is based on the hypothesis that pooled IVIG may result in the saturation of $\mathrm{Fc}$ receptors on phagocytes. Through competition, they prevent pathogenic autoantibodies to bind to these cells and prevent immune activation [18]. The beneficial effect of IVIG has been shown in several diseases with molecular mimicry as underlying pathophysiology. Marked improvement with this regimen is made in Guillain-Barré syndrome, chronic inflammatory demyelinating polyneuropathy and ITP [13, 31]. Doses vary from $0.4 \mathrm{~g} / \mathrm{kg} /$ day for 3-7 days in GuillainBarré to $1 \mathrm{~g} / \mathrm{kg}$ in single dose in ITP [19].

When scrutinising the literature, one randomised controlled clinical trial was found. Eighteen subjects were entered into this trial, which was designed to determine if IVIG or plasma exchange would be superior to prednisone in decreasing severity of chorea. Four patients were randomised to receive IVIG, eight received plasma exchange and six, prednisone. Mean chorea severity was significantly lower for the entire group at the 1-month follow-up evaluation, with an overall improvement in half of them. The between-group differences were not statistically significant due to small patient numbers. Nevertheless, clinical signs improved more rapidly and robustly in the IVIG and plasma exchange group than in the prednisone group [10].

\section{Conclusion}

SC is a rare but very disabling manifestation of ARF. Therapeutic treatment options are still in experimental stage. Based on pathophysiology and experiences in comparable diseases, IVIG seems a reasonable treatment option. This double case report on two SC patients indicates that IVIG is an effective treatment, although larger studies are needed to confirm this conclusion.

Conflict of interest All authors declare that they have no conflict of interest.

Open Access This article is distributed under the terms of the Creative Commons Attribution Noncommercial License which permits any noncommercial use, distribution, and reproduction in any medium, provided the original author(s) and source are credited.

\section{References}

1. Barash J, Margalith D, Matitiau A (2005) Corticosteroid treatment in patients with Sydenham's chorea. Pediatr Neurol 32(3):205-207 
2. Bronze MS, Dale JB (1993) Epitopes of streptococcal M proteins that evoke antibodies that cross-react with human brain. J Immunol 151:2820-2828

3. Carapetis JR, Currie BJ (1999) Rheumatic chorea in northern Australia: a clinical and epidemiological study. Arch Dis Child 80:353-358

4. Cardoso F (2008) Sydenham's chorea. Curr Treat Options Neurol 10(3):230-235

5. Cardoso F, Maia D, Cunningham MC, Valenca G (2003) Treatment of Sydenham chorea with corticosteroids. Mov Disord 18(11):1374-1377

6. Church AJ, Cardoso F, Dale RC et al (2002) Anti-basal ganglia antibodies in acute and persistent Sydenham's chorea. Neurology 59:227-231

7. de Queiroz Campos Araújo AP, Pádua PAB Filho HSM (2002) Management of rheumatic chorea. An observational study. Arch Neuropsychiatr 60(2-A):231-233

8. Demiroren K, Yavuz H, Cam L et al (2007) Sydenham's chorea: a clinical follow-up of 65 patients. J Child Neurol 22(5):550-554

9. Eshel G, Lahat E, Azizi E et al (1993) Chorea as a manifestation of rheumatic fever - a 30-year survey (1960-1990). Eur J Pediatr 152:645-646

10. Garvey MA, Snider LA, Leitman SF et al (2005) Treatment of Sydenham's chorea with intravenous immunoglobulin, plasma exchange, or prednisone. J Child Neurol 20(5):424-429

11. Genel F, Arslanoglu S, Uran N, Saylan B (2002) Sydenham's chorea: clinical findings and comparison of the efficacies of sodium valproate and carbamazepine regimens. Brain Develop 24:73-76

12. Husby G, van de Rijn I, Zabriskie B et al (1976) Antibodies reacting with cytoplasm of subthalamic and caudate nuclei neurons in chorea and acute rheumatic fever. J Exp Med 144:1094-1110

13. Jolles S, Sewell WAC, Misbach SA (2005) Clinical uses of intravenous immunoglobulin. Clin Exp Immunol 142:1-11

14. Kirvan CA, Cox CJ, Swedo SE, Cunningham MW (2007) Tubulin is a neuronal target of autoantibodies in Sydenham's chorea. J Immunol 178:7412-7421

15. Kirvan CA, Swedo SE, Heuser JS, Cunningham M (2003) Mimicry and autoantibody-mediated neuronal cell signalling in Sydenham chorea. Nat Med 9(7):914-920
16. Korn-lubetzki I, Brand A, Steiner I (2004) Recurrence of Sydenham chorea. Arch Neurol 61:1261-1264

17. Krisher K, Cunningham MW (1985) Myosin: a link between streptococci and heart. Science 227(4685):413-415

18. Lemieux R, Bazin R, Neron S (2005) Therapeutic immunoglobulins. Mol Immunol 42:839-848

19. Liumbruno G, Bennardello F, Lattanzio A et al (2009) Recommendations for the use of albumin and immunoglobulins. Blood Transfus 7:216-234

20. Martino D, Tanner A, Defazio G et al (2005) Tracing Sydenham's chorea: historical documents from a British paediatric hospital. Arch Dis Child 90:507-511

21. McMillan R (2000) Autoantibodies and auto antigens in chronic immune thrombocytopenic purpura. Semin Hematol 37(3):239-248

22. Paz JA, Silva CAA, Marques-Dias MJ (2005) Randomized double-blind study with prednisone in Sydenham's chorea. Pediatr Neurol 34(4):264-269

23. Peña J, Mora E, Cardoso J et al (2002) Comparison of the efficacy of carbamazepine, haloperidol and valproic acid in the treatment of children. Brain Develop 24:73-76

24. Special Writing Group of the Committee on Rheumatic Fever, Endocarditis, and Kawasaki Disease of the Council on Cardiovascular Disease in the Young of the American Heart Association (1992) Guidelines for the diagnosis of rheumatic fever. Jones Criteria 1992 update. JAMA 268(15):2069-2073

25. Stollerman GH (1997) Rheumatic fever. Lancet 349:935-942

26. Susuki K, Odaka M, Mori M et al (2004) Acute motor axonal neuropathy after mycoplasma infection. Evidence of molecular mimicry. Neurology 62:949-956

27. Taranta A, Stollerman GH (1956) The relationship of Sydenham's chorea to infection with group A streptococci. Am J Med 20:170

28. Teixeira AL Jr, Maia DP, Cardoso F (2005) Treatment of acute Sydenham's chorea with methyl-prednisolone pulse-therapy. Parkinsonism Relat Disord 11(5):327-330

29. Walker AR, Tani LY, Thompson JA et al (2007) Rheumatic chorea: relation to systemic manifestations and response to corticosteroids. J Pediatr 151:679-683

30. Weiner SG, Normandin PA (2007) Sydenham chorea. A case report and review of the literature. Pediatr Emerg Care 23(1):20-24

31. Weinstein R (2000) Therapeutic apheresis in neurological disorders. J Clin Apher 15:74-79 